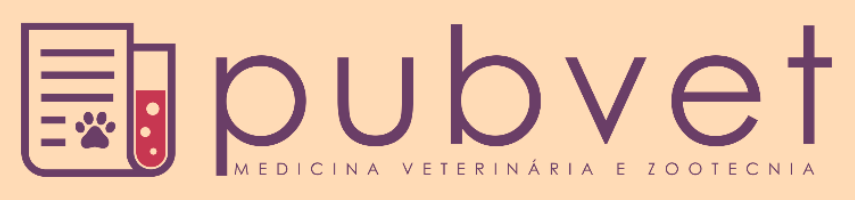

https://doi.org/10.31533/pubvet.v13n2a262.1-8

\title{
Uso da caça de subsistência no assentamento Santa Luzia, Cruzeiro do Sul, Acre
}

\author{
Anderson Barroso Damaceno ${ }^{1} \bullet$, Givanildo Pereira Ortega ${ }^{2} \bullet$, Luiz Carlos Batista Turci ${ }^{3 *}$ \\ ${ }^{1}$ Universidade Federal do Acre, Campus Floresta (Bacharel em Engenharia Florestal), Cruzeiro do Sul, Acre, Brasil. \\ ${ }^{2}$ Professor da Universidade Federal do Acre, Campus Floresta (Laboratório de Recursos Florestais e Conservação da Natureza), Cruzeiro do Sul-AC, Brasil. \\ ${ }^{3}$ Professor da Universidade Federal do Acre, Campus Floresta (Laboratório de Herpetologia), Cruzeiro do Sul-AC, Brasil. \\ * Autor para correspondência: luizcarlosturci@gmail.com
}

\begin{abstract}
Resumo. Na Amazônia, a fauna nativa é considerada a maior fonte de proteína animal consumida pelas populações rurais e povos tradicionais. As principais espécies cinegéticas utilizadas por essas comunidades destacam-se, grandes mamíferos (ungulados: queixadas, veados e antas; primatas e roedores como as pacas e as cutias), avifauna (mutuns, jacamins, jacus e nambus) e os répteis (quelônios). O presente estudo caracteriza o uso da caça de subsistência pelos moradores rurais no assentamento Santa Luzia, em Cruzeiro do Sul, Acre. A coleta de dados deu-se por meio de questionário semiestruturado, sendo composto por perguntas fechadas e abertas, um total de 101 famílias foram entrevistadas. Cerca de 163 animais são abatidos a cada mês, totalizando aproximadamente 1400 animais abatidos anualmente. Foram apontadas 11 espécies de uso cinegéticos, destas o tatu (Dasypus novemcinctus), jacu (Opisthocomus hoazin), paca (Cuniculus paca) e o veado (Mazama sp.) são as mais visadas para caça na localidade, tendo maior aceitação pelos caçadores. A técnica de caça a ponto é a mais utilizada pelos caçadores seguida pela caça com cachorros. Atualmente, o tempo gasto na caçada circunda em torno de 6 a 16 horas para obter um animal. Estudos mais aprofundados voltados ao entendimento da pressão da caça sobre essas espécies se tornam de suma importância para manutenção e conservação da fauna local.
\end{abstract}

Palavras chave: amazônia, caça de subsistência, fauna cinegética

\section{The use of subsistence hunting at Santa Luzia settlement, in Cruzeiro do Sul, Acre}

\begin{abstract}
In Amazon, native fauna is considered the major source of animal protein consumed by rural populations and traditional people. The main cinegetic species used by these communities stand out: large mammals (ungulates: white-lipped peccary, deer and tapir; primates and rodents such as pacas and agoutis), avifauna (curassows, jacamins, jacus and nambus) and the reptiles (chelonians). The present study characterizes the use of subsistence hunting by rural dwellers at Santa Luzia settlement, in Cruzeiro do Sul, Acre. The data collection was made by semi-structured questionnaire, composed of closed and open-ended questions, a total of 101 families were interviewed. About 163 animals where slaughtered each month at the study area, totaling approximately 1.400 animals slaughtered annually. Were pointed out 11 species of cinegetic use, among them, the armadillo (Dasypus novemcinctus), jacu (Opisthocomus hoazin), paca (Cuniculus paca) and the deer (Mazama sp.) are the most targeted for hunting in the locality, having the major acceptance by hunters. The technique of hunting on the spot is the most used by hunters followed by hunting with dogs. Nowadays, the time spent in the hunt is around 6 to 16 hours to obtain
\end{abstract}


an animal. Deeper studies about understanding the pressure of hunting over these species become highly important to their maintenance and conservation on site.

Keywords: cinegetic fauna, subsistence hunting, Amazon

\title{
El uso de la caza de subsistencia en el asentamiento Santa Luzia, Cruzeiro do Sul, Acre
}

\begin{abstract}
Resumen. En la Amazonia, la fauna nativa es considerada la mayor fuente de proteína animal consumida por las poblaciones rurales y pueblos tradicionales. Entre las principales especies, de fuentes proteicas, utilizadas por estos pueblos destacamos los grandes mamíferos ungulados: (Pécari de collar, ciervos y antas, primates y roedores como las pacas y las cutías), avifauna (mutuns, jacamines, jacus y nambus) y los reptiles (quelonios). El presente estudio caracteriza el uso de la caza de subsistencia por los habitantes rurales en el asentamiento Santa Luzia, en Cruzeiro do Sul, Acre. La recolección de datos se dio por medio de un cuestionario semiestructurado, compuesto por preguntas cerradas y abiertas, un total de 101 familias fueron entrevistadas. Cerca de 163 animales se sacrifican cada mes en el área de estudio, totalizando aproximadamente 1400 animales sacrificados anualmente. De las especies utilizadas como fuente de proteínas por estos pueblos fueron destacadas 11, cuyo el uso tornase imprescindible en su cotidiano, de las cuales el armadillo (Dasypus novemcinctus), jacu (Opisthocomus hoazin), paca (Cuniculus paca) y el venado (Mazama sp.) Son los más visados para caza en la localidad, teniendo mayor aceptación por los cazadores. La técnica de caza a punto es la más utilizada por los cazadores seguida por la caza con perros. Actualmente, el tiempo dedicado a la cacería circunda alrededor de 6 a 16 horas para obtener un animal. Los estudios más profundos dirigidos al entendimiento de la presión de la caza sobre esas especies se vuelven de suma importancia para el mantenimiento y la conservación de la fauna local.
\end{abstract}

Palabras clave: amazonia, caza de subsistencia, fauna cinegética

\section{Introdução}

O Brasil compõe o grupo dos países megadiversos e a Amazônia possui a maior biodiversidade do planeta, apresentando uma grande riqueza de mamíferos, aproximadamente 400 espécies, sendo 231 endêmicas (Mittermeier et al., 1988; Paglia et al., 2012). Dentre os animais, os vertebrados são o grupo que têm maior interação com os seres humanos (Mendonça et al., 2011; Wilson, 1999).

Na Amazônia, a fauna nativa é considerada a maior fonte de proteína animal consumida pelas populações rurais e povos tradicionais (Peres, 2000; Redford, 1997; Robinson \& Bennett, 1999). As principais espécies cinegéticas utilizadas por essas comunidades destacam-se, grandes mamíferos (ungulados: queixadas, veados e antas; primatas e roedores como as pacas e as cutias), avifauna (mutuns, jacamins, jacus e nambus) e os répteis (quelônios) (Redford, 1997). Segundo Alves et al. (2012) o uso da caça não só atende ao apelo de subsistência, mas também apresenta valor cultural e econômico, onde aquisição da proteína animal fornece matéria prima para produção de subprodutos e uso zooterápico para as famílias tradicionais.

Discussões sobre capacidade de suporte dos recursos faunísticos no local, ações de monitoramento dos impactos sobre as populações cinegéticas, elaboração de calendários de caça, são fundamentais para implementação de um manejo participativo envolvendo a população e os gestores das comunidades rurais e povos tradicionais, com objetivo de adotar medidas mitigatórias aos possíveis impactos atribuídos à caça de subsistência, tais como, a escolha de áreas prioritárias e a rotatividade dos locais de caça, escolha das técnicas de caça menos impactantes e a adoção de medidas de fiscalização (Townsend, 2004).

O presente estudo caracteriza o uso da caça de subsistência pelos moradores rurais no assentamento Santa Luzia, em Cruzeiro do Sul, Acre. 


\section{Material e métodos}

O estudo foi realizado no assentamento Santa Luzia, cuja área é de 62.267,88 hectares, havendo 879 famílias assentadas, está localizado no ramal 03 ( $\left.8^{\circ} 01^{\prime} 52^{\prime} \mathrm{S}, 72^{\circ} 35^{\prime} 09^{\prime} \mathrm{W}\right)$ (Figura 1), no município de Cruzeiro do Sul, Acre, cuja área é de $8.779,2$ km², e população de 78.507 habitantes.

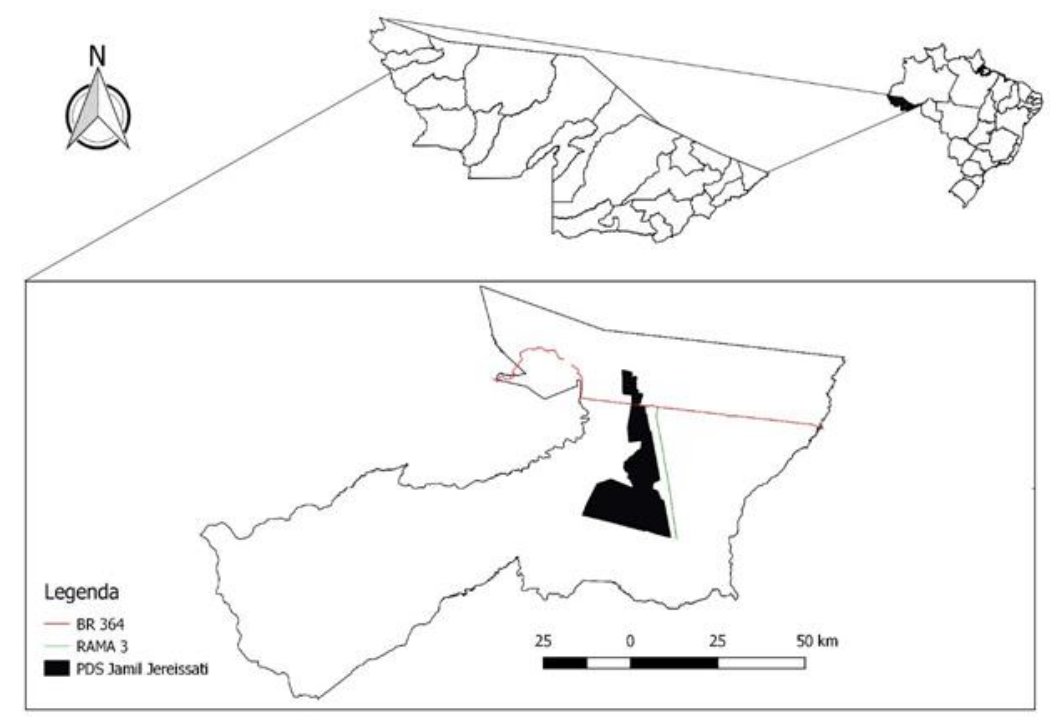

Figura 1. Localização do assentamento Santa Luzia, Cruzeiro do Sul, Acre.

A coleta de dados deu-se por meio de questionário semiestruturado composto por perguntas fechadas e abertas. Todos entrevistados foram convidados a assinar o TCLE - termo de consentimento livre e esclarecido para participação na pesquisa. As entrevistas foram realizadas entre o período de outubro de 2017 a fevereiro de 2018. Objetivando obter informações em torno dos aspectos socioeconômicos para caracterização do perfil dos caçadores, identificação dos animais abatidos, pressão de caça e os métodos de caça utilizados.

Os resultados foram organizados de forma descritiva, agrupados por categorias temáticas, na qual os dados foram organizados com base nas questões realizadas. Segundo Bardin (2004) as categorias constituem um meio de classificar os dados descritivos que foram recolhidos, de forma que o material de um determinado tópico possa ser apartado dos outros dados, facilitando a interpretação.

\section{Resultados e discussão}

Foram entrevistadas 101 famílias, distribuídas em cerca de 26 quilômetros na área do assentamento. Aproximadamente 50\% das famílias residem no assentamento entre 11 e 20 anos. Foi percebida uma alta dependência da caça para subsistência no local. Cerca de 60\% dos entrevistados agregam uma renda em torno de 1 a 2 salários com auxílios do governo (aposentadorias e demais benefícios). A renda observada no assentamento é semelhante, quando comparadas com outras comunidades amazônicas, sendo caracterizadas de baixo nível de renda, fazendo-se necessárias ações de políticas públicas visando melhor desenvolvimento socioambiental da comunidade (Santiago, 2016). Em estudo realizado por Ribeiro et al. (2007), em sete comunidades em Belém-PA, caracterizadas como sendo de baixa renda, constatou-se a venda e comércio ilegal da fauna silvestre como fonte de renda por parte dos moradores, a fim de suprir as despesas familiares.

Quanto ao nível de escolaridade, $31 \%$ dos pais e $19 \%$ das mães são analfabetos, a maior parte dos entrevistados haviam concluído o nível fundamental. A maior parte dos pais $(\mathrm{n}=59-58.4 \%)$ ocupa a função de agricultor e as mães com a função de dona da casa $(n=54-53.4 \%)$. Dados semelhantes são apresentados em outras localidades amazônicas, demonstrando a carência educacional nestas comunidades estudadas (Santiago, 2016). Miranda \& Alencar (2007), em estudos na caatinga, constataram que a maioria dos caçadores exercia a função de agricultor, e a prática da caça era realizada para aquisição de proteína animal. Fuccio et al. (2003), em estudos na região de Rio Branco-AC, observaram que os caçadores exerciam profissões de diaristas, agricultores, seringueiros e comerciantes, 
sendo maioria semialfabetizados. Na reserva de desenvolvimento sustentável Amanã (RDS, Amanã) no Amazonas, moradores de quatro comunidades pesquisadas, obtinham sua renda principalmente a partir da produção da farinha de mandioca (Valsecchi \& Amaral, 2009), fato também observado no presente estudo. A maior parte dos entrevistados $(60,4 \%)$ depende parcialmente da caça para obtenção de alimento. A periodicidade da caça ocorre em média três vezes ao mês, sendo abatidos cerca de dois animais por caçada. Valsecchi \& Amaral (2009) observaram periodicidade semelhante, variando de uma a cinco caçadas ao mês. Rosas \& Drumond (2007), em estudos realizados em dois seringais no Estado do Acre, observaram que a caça ocorria em torno de 1 a 60 dias.

As espécies cinegéticas registradas são pertencentes a duas classes, mammalia (mastofauna) e aves (avifauna). Cerca de 163 animais são abatidos a cada mês, totalizando cerca de 1.400 animais anualmente. Entre os mamíferos, os espécimes mais caçados, são os tatus-galinha (Dasypus novemcinctus), pacas (Cuniculus paca) e veados (Mazama sp.). Dentre as aves o jacu-cigano (Opisthocomus hoazin) foi a única espécie mencionada no local (Tabela 1).

Tabela 1. Animais abatidos no Assentamento Santa Luzia, no Município de Cruzeiro do Sul - AC.

\begin{tabular}{llc}
\hline Classe / Animal & Espécie & Animais Abatidos \\
\hline Mammalia & & 41 \\
Tatu-galinha & Dasypus novemcinctus (Linnaeus, 1758) & 26 \\
Paca & Cuniculus paca (Linnaeus, 1766) & 17 \\
Veado & Mazama sp. & 15 \\
Queixada & Tayassu pecari (Link, 1795) & 13 \\
Cateto & Pecari tajacu (Linnaeus, 1758) & 13 \\
Cutia & Dasyprocta aguti (Linnaeus, 1766) & 3 \\
Guariba & Alouatta caraya (Humboldt, 1812) & 1 \\
Porco-Espinho & Coendou prehensilis (Linnaeus, 1758) & 1 \\
Tamanduá & Tamandua tetradactyla (Linnaeus, 1758) & 1 \\
Mucura & Didelphis marsupialis (Linnaeus, 1758) & 32 \\
Aves & & 163 \\
Jacu-cigano & Opisthocomus hoazin (Muller, 1776) & \\
\hline Total & &
\end{tabular}

Em comunidades rurais e povos tradicionais na região amazônica observar-se certa semelhança na preferência das espécies cinegéticas durante a caça, fato esse pode estar relacionado a fatores como a abundância da espécie na localidade, grau de dificuldade e custo da caça, e o sabor e rendimento de carne fornecido pelo animal. Onde as práticas da caça são direcionadas em sua maior parte para os mamíferos de médio e grande porte (Cullen et al., 2001).

Estudos conduzidos por Rosas \& Drumond (2007), em dois seringais no Estado do Acre, apontaram preferências de caça semelhantes ao observado no presente estudo, sendo o veado-capoeiro (Mazama americana) como a caça preferida em ambos locais, seguido pela cutia (Dasyprocta sp.), paca (Agouti paca), caititu (Pecari tajacu) e tatus (Dasypodidae). Almeida et al. (2012) apontaram que a paca (C. paca) é o animal de maior preferência para a caça, seguido pela cutia e tatus no Parque Nacional Serra do Divisor (PNSD), no Alto Juruá, Acre. Na RDS, Amanã, no estado do Amazonas, cerca de 373 animais são abatidos mensalmente, sendo os mamíferos, aves e répteis os grupos mais visados, dentre as espécies mais consumidas o caititu (T. pecari) e a paca (C. paca) são as espécies de maior preferência para consumo (Valsecchi \& Amaral, 2009). Ribeiro et al. (2007), em estudo sobre uso dos recursos naturais por populações em assentamentos do INCRA, também registrou a (paca, cutia, tatu, caititu e o veado) dentre as espécies mais caçadas. Dentre os onze animais citados para o consumo, o tatu-galinha (Dasypus novemcinctus), jacu (Opisthocomus hoazin) e a paca (Cuniculus paca) são as espécies com maior aceitação e o guariba (Alouatta caraya) e a mucura (Didelphis marsupialis) sendo as espécies com maior rejeição pelos caçadores na localidade (Figura 2).

Em estudo realizado na Área de Proteção Ambiental Guaraqueçaba, observaram que os tatus, paca e jacus também foram as espécies mais visadas pelos caçadores (Andriguetto-Filho et al., 1998). O tatugalinha (D. novemcinctus) foi a espécie mais abatida $(\mathrm{n}=41)$ e de maior aceitação no presente estudo. Essa espécie também foi apontada sendo a de maior preferência em estudo feito nas comunidades indígenas "Tupinambá de Olivença" na Bahia (Pereira \& Schiavetti, 2010). Esse fato pode estar 
relacionado pela facilidade de encontro deste animal tanto em ambientes abertos e capoeiras na localidade, o baixo custo da caçada e pelo sabor da sua carne, segundo relatos dos moradores na área de estudo. O tatu é uma espécie de hábitos crepusculares e noturnos, habitam uma ampla variedade de ambientes, apresentando uma dieta generalista (Pereira \& Schiavetti, 2010). Isso faz com que este animal possa ser observado em diferentes locais, como áreas de vegetação aberta, capoeiras, pastagem (Costa et al., 2005), ambientes estes predominantes na área do estudo.

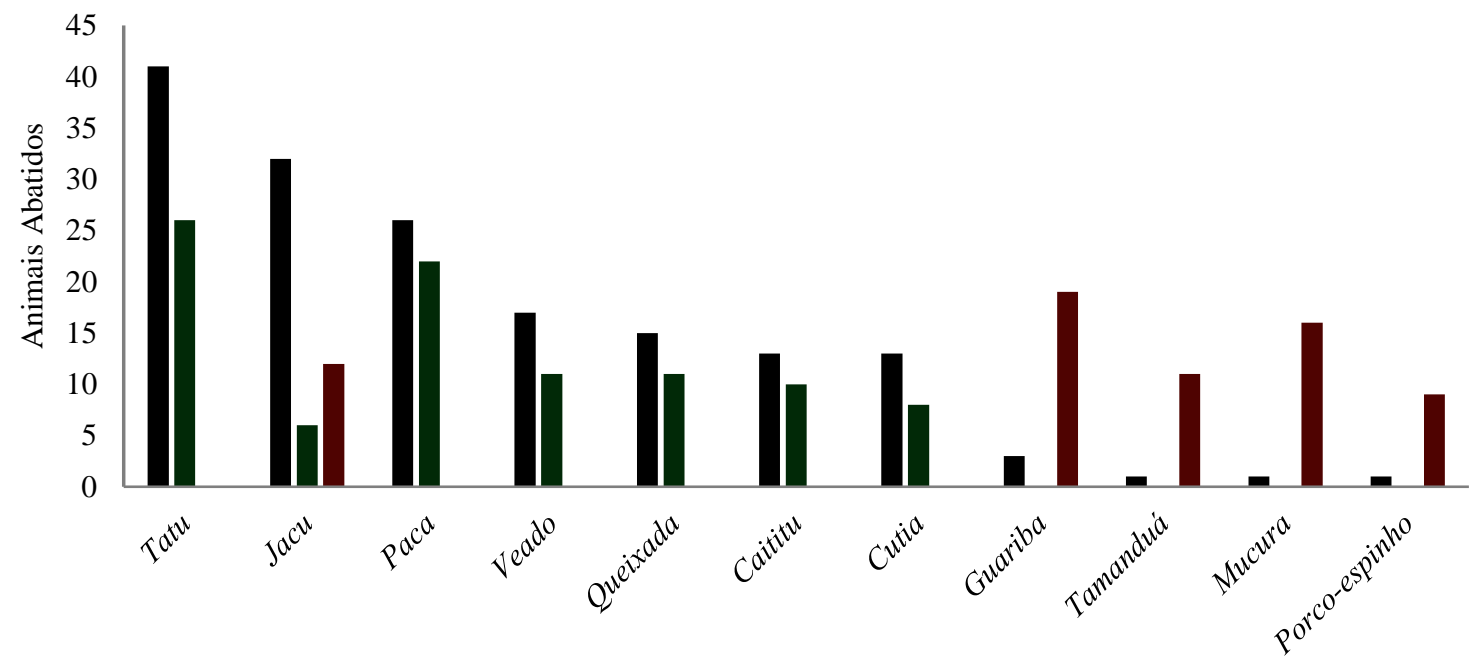

Figura 2. Número mensal de animais caçados (coluna preta), aceitação (coluna verde) e rejeição (coluna vermelha) no Assentamento Santa Luzia, Cruzeiro do Sul, Acre.

A paca (C. paca) está entre os animais mais abatidos $(n=26)$ e de maior aceitação neste estudo. Essa grande aceitação também é observada em demais estudos em comunidades de caçadores rurais, populações tradicionais e indígenas na região amazônica, devido ao sabor de sua carne (Almeida et al., 2012; Pereira \& Schiavetti, 2010; Van Vliet et al., 2014). Segundo Moreira \& Macdonald (1997), os roedores é a segunda ordem de mamífero mais caçado em comunidades tradicionais. Deutsch \& Puglia (1990) mencionam que a paca é uma espécie historicamente caçada, fato esse associado ao sabor de sua carne.

As maiores rejeições foram referidas ao guariba (A. caraya) e a mucura (D. marsupialis). A maior parte dos entrevistados relatou como sendo "nojento" e sem necessidade do abate. Van Vliet et al. (2014) observaram a rejeição da caça de macacos e de certas aves em estudos nas comunidades que fazem fronteira com o Brasil. Valsecchi \& Amaral (2009) relataram poucos abates voltados a espécies de primatas.

Pode-se verificar que os caçadores no presente estudo fazem uso de diferentes técnicas de caça para captura dos animais. A caça a ponto é a mais utilizada pelos caçadores, seguida pela caça com cachorros (Figura 3). Pode-se observar que os caçadores, no presente estudo, caçam os animais mais ativamente, diferentemente dos caçadores indígenas na Bahia, onde a técnica mais empregada é a armadilha. Ainda, os caçadores apresentaram uma maior diversificação de técnicas, um total de 10 tipos diferentes de estratégias de captura de animais, demostrando um maior preparo e conhecimento na captura da fauna silvestre (Pereira \& Schiavetti, 2010). Em outros estudos realizados em seringais no Estado do Acre, a caça de espera foi a mais praticada pelos entrevistados (Calouro \& Marinho-Filho, 2005; Rosas \& Drumond, 2007). No presente estudo a caça de espera foi a menos praticada pelos caçadores no assentamento. Analisando os aspectos da caça com cães em uma região do semiárido no Nordeste, onde $81 \%$ dos caçadores utilizam o cão como auxiliar ou como principal meio de busca/captura dos recursos faunísticos (Vasconcelos Neto et al., 2012).

Importante ressaltar a não aceitação de parte dos caçadores locais, aos que utilizam a técnica de caça com cachorros, onde estes afirmavam que a utilização dessa técnica ocasiona a redução dos animais nas proximidades do local. Fato esse, foi apontado em estudo conduzido em dois seringais no Acre, onde 
foi restringida a caça com cachorro, por ser altamente seletiva e por afugentar a caça da localidade (Calouro \& Marinho-Filho, 2005).

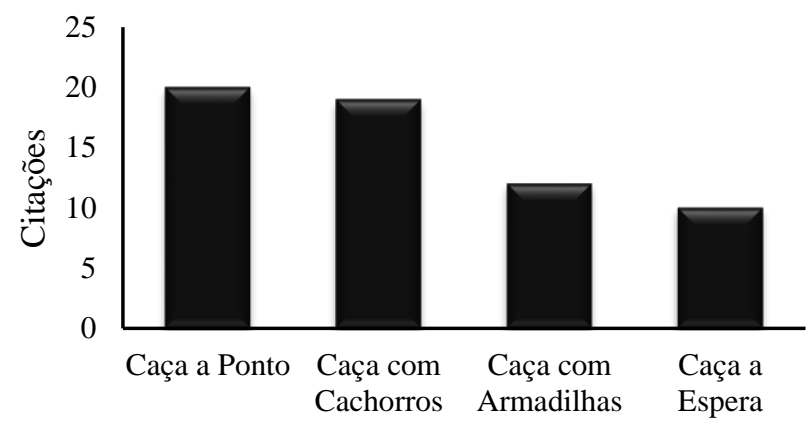

Figura 3. Técnicas de caça empregadas pelos caçadores no Assentamento Santa Luzia

De acordo com os caçadores locais $(n=56)$, foi percebido que ao passar dos anos houve a diminuição na abundância de animais nas proximidades das residências, onde o abate da caça era feito com menor tempo e percorrido distâncias menores. Atualmente, o tempo gasto na caçada circunda em torno de 6 a 16 horas para obter o caça. O tempo gasto para obtenção da caça no presente estudo é considerado alto, quando comparado a outros estudos no Acre (Calouro \& Marinho-Filho, 2005; Rosas \& Drumond, 2007). Almeida et al. (2012) constataram que por parte dos caçadores, houve uma diminuição na visualização dos animais. Esse fato pode indicar uma diminuição na densidade dos indivíduos devido um alto grau de pressão sobre a espécies cinegéticas no local. Estratégias de rotatividade de locais de caça pode ser uma alternativa para diminuir a pressão de caça local.

Em estudo feito por Vieira (2013) observou a rotatividade da caça feita pelos caçadores na RDS Piagaçu-Purus no Amazonas com o intuito de amezinhar a pressão de caça nos locais mapeadas para atividades de caça. Miranda et al. (2002) citam animais como a paca (Cuniculus paca), porco-do-mato (Tayassu tajacu) e Cutia (Dasyprocta sp.), como sendo espécies resistentes a pressão de caça. No entanto, estudos mais aprofundados são necessários para entender o grau de pressão da caça no local e propor medidas que visão minimizar os impactos relacionados caça de subsistência.

\section{Conclusão}

Foi possível detectar parte dos caçadores ativos no Assentamento Santa Luzia. A caça é exercida como um complemento nutricional na aquisição de proteína animal. Oito espécies cinegéticas são mais visadas nas caçadas. O tatu, jacu, paca, veado e o caititu são as espécies de maior aceitação para o consumo. Estudos mais aprofundados voltados ao entendimento da pressão da caça sobre essas espécies se tornam de suma importância para manutenção e conservação da fauna cinegética local.

Como sugestão, a fim de minimizar o impacto da caça sobre as espécies da fauna silvestre no assentamento Santa Luzia, propõe-se a implementação de ações de políticas públicas governamentais, incentivando a criação de animais domesticados em cativeiro como (frangos), criatórios de peixes (tambaquis entre outros) e até mesmo a criação de animais silvestres em cativeiro, essas atividades propiciam um melhor uso da área, facilitam a obtenção de proteína animal, podem atuar como uma fonte de renda alternativa para as famílias envolvidas, estimulando o desenvolvimento sustentável no local e preservação dos recursos faunísticos.

\section{Referências bibliográficas}

Almeida, G. S., Souza, P. O., Souza, A. M., Souza, C. R. \& Oliveira, K. A. (2012). Percepção das populações do interior e do entorno do parque nacional serra do divisor - acre sobre a caça cinegética e seus efeitos sobre a abundância dos recursos faunísticos. Enciclopédia Biosfera, Centro Científico Conhecer, 81902-1912.

Alves, R. R. N., Gonçalves, M. B. R. \& Vieira, W. L. S. (2012). Caça, uso e conservação de vertebrados no semiárido Brasileiro. Tropical Conservation Science, 5(3):394-416. 
Andriguetto-Filho, J. M., Krüger, A. C. \& Lange, M. B. R. (1998). Caça, biodiversidade e gestão ambiental na Área de Proteção Ambiental de Guaraqueçaba, Paraná, Brasil. Biotemas, 11(2):133156.

Bardin, L. (2004). Análise de conteúdo. São Paulo, Brasil.

Calouro, A. M. \& Marinho-Filho, J. S. (2005). A sustentabilidade da caça de subsistência entre seringueiros do Acre (Brasil). In P. M. Drumond (Ed.), Fauna do Acre. Rio Branco, Acre: Edufac.

Costa, L. P., Leite, Y. L. R., Mendes, S. L. \& Ditchfield, A. D. (2005). Mammal conservation in Brazil. Conservation Biology, 19(3):672-679.

Cullen, L., Bodmer, E. R. \& Valladares-Padua, C. (2001). Ecological consequences of hunting in Atlantic forest patches, São Paulo, Brazil. Oryx, 35(2):137-144.

Deutsch, L. A. \& Puglia, L. R. R. (1990). Os animais silvestres: proteção, doenças e manejo. São Paulo, Brasil: Globo.

Fuccio, H., Carvalho, E. F. \& Vargas, G. (2003). Perfil da caça e dos caçadores no Estado do Acre, Brasil. Revista Aportes Andinos, 61-18.

Mendonça, L. E. T., Souto, C. M., Andrelino, L. L., Souto, W. M. S., Vieira, W. L. S. \& Alves, R. R. N. (2011). Conflitos entre pessoas e animais silvestres no semiárido paraibano e suas implicações para conservação. Sitientibus Série Ciências Biológicas, 11185-199.

Miranda, C. L. \& Alencar, G. S. (2007). Aspectos da atividade de caça no Parque Nacional Serra da Capivara, estado do Piauí, Brasil. Natureza \& Conservação, 5(1):27-34.

Miranda, J. R., Nunes, V. S. \& Souza, M. F. B. (2002). Extrativismo animal em zona de fronteira agrícola na Amazônia: o caso do Município Machadinho d' Oeste-RO. Embrapa Monitoramento por Satélite, 161-36.

Mittermeier, R. A., Rylands, A. B., Coimbra-Filho, A. F. \& Fonseca, G. A. B. (1988). Systematics: species and subspecies-an update. In R. A. Mittermeier, A. B. Rylands, A. F. Coimbra-Filho \& G. A. B. Fonseca (Eds.), Ecology and behaviour of Neotropical Primates (Vol. 2, pp. 13-75). Rio de Janeiro, Brasil: Academia Brasileira de Ciência.

Moreira, J. R. \& Macdonald, D. W. (1997). Técnicas de manejo de capivaras e outros grandes roedores na Amazônia. In C. Valladares-Pádua \& R. E. Bodmer (Eds.), Manejo e conservação de vida silvestre no Brasil. Brasília, Brasil: Conselho Nacional de Desenvolvimento Científico e Tecnológico.

Paglia, P. A., F., G. A. B., Rylands, A. B., Herrmann, G., Aguiar, S. M. L., Chiarello, G. A., . . Patton, L. J. (2012). Lista Anotada dos Mamíferos do Brasil. Occasional Papers in Conservation Biology, 61-76.

Pereira, J. P. R. \& Schiavetti, A. (2010). Conhecimentos e usos da fauna cinegética pelos caçadores indígenas" Tupinambá de Olivença. Biota Neotropica, 10(1):175-183.

Peres, C. A. (2000). Effects of subsistence hunting on vertebrate community structure in Amazonian forests. Conservation Biology, 14(1):240-253.

Redford, K. H. (1997). A floresta vazia. In C. Valladares-Pádua \& R. E. Bodmer (Eds.), Manejo e conservação da vida silvestre. Belém, Pará: Sociedade Civil Mamirauá.

Ribeiro, A. S. S., Palha, M. D. C., Tourinho, M. M., Whiteman, C. \& Silva, A. S. L. (2007). Utilização dos recursos naturais por comunidades humanas do Parque Ecoturístico do Guamá, Belém, Pará. Acta Amazônica, 37(2):235-240.

Robinson, J. G. \& Bennett, E. L. (1999). Carrying capacity limits to sustainable hunting in tropical forests. In J. G. Robinson \& E. L. Bennett (Eds.), Hunting for sustainability in tropical forests. New York-EUA: Columbia University Press.

Rosas, G. K. C. \& Drumond, P. M. (2007). Caracterização da caça de subsistência em dois seringais localizados no Estado do Acre (Amazônia, Brasil). Embrapa Acre-Documentos, 109(1):1-31.

Santiago, D. R. (2016). Perfil socioeconômico e ambiental das comunidades tradicionais amazônicas: $\mathrm{O}$ caso das comunidades limitantes à área de Manejo florestal da Precious Wood Amazon. Revista Perspectivas do Desenvolvimento, 4(5):1-30. 
Townsend, W. R. (2004). Increasing local stakehold participation in wildlife management projects with rural communities. In K. M. Silvius, R. E. Bodmer \& J. M. V. Fragoso (Eds.), People in Nature. New York, USA: Columbia University Press.

Valsecchi, J. \& Amaral, P. V. (2009). Perfil da caça e dos caçadores na reserva de desenvolvimento sustentável Amanã, Amazonas - Brasil. Scientific Magazine UAKARI, 5(2):33-48.

Van Vliet, N., Quiceno-Mesa, M. P., Cruz-Antia, D. \& Yagüe, B. (2014). Carne de caça e segurança alimentar na zona da tríplice fronteira amazônica (Colômbia, Peru e Brasil). Bogotá, Colômbia: Fundação Omacha.

Vasconcelos Neto, C. F. A., Santos, S. S., Sousa, R. F., Fernandes-Ferreira, H. \& Lucena, R. F. P. (2012). A caça com cães (Canis lupus familiaris) em uma região do semiárido do nordeste do Brasil. Biofar, 1, 1-16. Biofar, 11-16.

Vieira, M. A. R. M. 2013. Influências dos sistemas de manejo formal e informal na atividade de caça de subsistência na RDS Piagaçu-Purus, AM. Dissertação (Mestrado), INPA, Manaus-AM.

Wilson, E. O. (1999). A situação atual da diversidade biológica. Rio de Janeiro, Brasil: Nova Fronteira.

Recebido: 23 de dezembro, 2018 .

Aprovado: 5 janeiro, 2018

Publicado: 15 fevereiro, 2019

Licenciamento: Este artigo é publicado na modalidade Acesso Aberto sob a licença Creative Commons Atribuição 4.0 (CC-BY 4.0), a qual permite uso irrestrito, distribuição, reprodução em qualquer meio, desde que o autor e a fonte sejam devidamente creditados. 